Research report

\title{
Depression, anxiety and cardiovascular disease: Which symptoms are associated with increased risk in community dwelling older adults?
}

\author{
Damien Gallagher ${ }^{\mathrm{a}, *, 1}$, Claire O’Regan ${ }^{\mathrm{b}, 1}$, George M. Savva ${ }^{\mathrm{b}}$, Hillary Cronin ${ }^{\mathrm{b}}$, \\ Brian A. Lawlor ${ }^{\mathrm{c}}$, Rose A. Kenny ${ }^{\mathrm{b}}$ \\ ${ }^{a}$ Cluain Mhuire Mental Health Services, Blackrock, Co. Dublin, Ireland \\ b Department of Medical Gerontology, Trinity College, Dublin, Ireland \\ ' Mercer's Institute for Research on Ageing, St James's Hospital, Dublin, Ireland
}

\section{A R T I C L E I N F O}

Article history:

Received 23 January 2012

Received in revised form

18 April 2012

Accepted 19 April 2012

Keywords:

Depression

Anxiety

Cardiovascular disease

\begin{abstract}
A B S T R A C T
Background: Depression is a risk factor for Cardiovascular Disease (CVD). It has been reported that somatic symptoms of depression and not cognitive symptoms are associated with increased risk although findings have been inconsistent. Few studies have examined whether co-morbid anxiety confers additive risk.

Methods: We conducted a cross sectional analysis of 7872 community dwelling adults aged 50 years and over from The Irish LongituDinal Study on Ageing (TILDA). Depressive and anxiety symptoms were assessed with Center for Epidemiologic Studies Depression (CES-D) scale and the anxiety subscale of the Hospital Anxiety and Depression Scale (HADS-A), respectively. We conducted logistic regression analyses to determine the relationship between depression, anxiety, individual depressive symptoms and CVD. We further determined whether co-morbid anxiety was associated with increased risk. Results: Seven hundred and thirty eight (9.4\%) study participants reported clinically significant depression. Depression was associated with 80\% increased risk of CVD following adjustment for cardiovascular risk factors. Individual depressive symptoms most consistently associated with CVD included low mood, sadness, amotivation, fatigue, diminished appetite and concentration difficulties. Anxiety was associated with increased risk of CVD but did not confer additive risk in participants with depression.

Limitations: Cross sectional design.

Conclusion: Core symptoms of depression, which are both cognitive and somatic in nature, are associated with increased risk of CVD while co-morbid anxiety did not confer additive risk. It is important that clinicians give due regard both to both cognitive and somatic symptoms of depression when determining cardiovascular risk. Future longitudinal investigation should confirm these findings and explore potential pathological mechanisms.
\end{abstract}

(c) 2012 Elsevier B.V. All rights reserved.

\section{Introduction}

Depression has been associated with both incident cardiovascular disease (CVD) and increased mortality following myocardial infarction (Barth et al., 2004; Rugulies, 2002). These effects appear to be independent of other known cardiovascular risk factors. A number of pathophysiological and behavioural mechanisms have been proposed including adverse health behaviours, stress axis activation, inflammatory activation and changes in autonomic function (Joynt et al., 2003). A small number of interventional studies in patients with CVD have demonstrated small

\footnotetext{
* Corresponding author. Tel: +35312172100; fax: + 35312833886

E-mail address: gallagherdamien@hotmail.com (D. Gallagher).

1 These authors contributed equally to this work.
}

improvements in depression with no improvement in cardiac outcomes (Gallagher, 2007). One possibility for these negative findings may be a failure to identify patients at greater risk and intervene effectively at a sufficiently early stage. There is, therefore, increased interest in understanding if particular depressive symptoms are associated with greater cardiovascular risk than others (Frasure-Smith and Lesperance, 2010).

Recently, it has been reported that somatic symptoms of depression are more closely associated with cardiovascular risk than cognitive symptoms (De Jonge et al., 2006). Conversely it has been reported that cognitive symptoms may be more "cardiotoxic" (Barefoot et al., 2000). One potential reason for these conflicting findings is that most studies to date have used factor analyses, thereby clumping symptoms into groups to determine which group is more closely associated with cardiovascular outcomes. This gives us relatively limited information about the importance of individual 
symptoms. It is possible for a symptom to be alternately assigned to a "somatic" or "affective" factor depending on the nature of the instrument used and the factor analysis conducted (De Jonge et al., 2006; Barefoot et al., 2000). A symptom level analysis would provide increased clarity in this regard and would also allow for comparison of individual symptoms across studies which have used different diagnostic instruments. One prospective symptom level analysis in outpatients with stable coronary heart disease reported that somatic depressive symptoms were independently predictive of further cardiovascular events while cognitive symptoms were not (Hoen et al., 2010). It is known that certain cognitive depressive symptoms may be reported less frequently by older adults (Brodaty et al., 2005) and investigators in this study noted that that insufficient power may have accounted for the negative findings in this instance (Hoen et al., 2010). Another study in a relatively small sample of community dwelling older adults with depression failed to find any association between individual symptoms and CVD (Licht-Strunk et al., 2004). The strength of the association between somatic depressive symptoms and CVD has led to speculation that the association between depression and CVD may be secondary to residual confounding from co-morbid physical conditions. However, analyses to date, provide little or only partial support for this hypothesis given that the association persists following adjustment for physical co-morbidity (De Jonge et al., 2006; Hoen et al., 2010). Given that symptoms ordinarily considered to be cognitive or somatic frequently co-occur and that there is considerable evidence to suggest that such symptoms exist along a continuum (Kessing, 2007), it might be anticipated that both components of the depressive syndrome would be associated to some degree with adverse cardiovascular outcomes. This observation would decrease the likelihood that the association between somatic depressive symptoms and CVD is simply secondary to residual confounding from co-morbid physical conditions. It has also been reported that positive affect may protect against incident coronary heart disease (Davidson et al., 2010). The Center for Epidemiologic Studies Depression (CES-D) scale differs from other measures in that it contains a number of items relating to positive affect and interpersonal functioning (Radloff, 1977). To the best of our knowledge the relationship between these items and CVD has not previously been examined. Similarly, the role of anxiety symptoms in depression is not fully understood. Anxiety is frequently co-morbid with depression and few studies have examined whether co-morbid anxiety is associated with increased risk of CVD. One previous study concluded that patients with co-morbid anxiety and depression were not at increased risk of CVD than those with either alone (Frasure-Smith and Lesperance, 2008). Conversely it has been reported that comorbid anxiety may account for most of the association between depression and CVD (Vogelzangs et al., 2010).

We therefore undertook to examine the relationship between individual depressive symptoms and CVD in a larger sample than previously described. We hypothesised that in this larger sample; there would be a significant relationship between both cognitive and somatic depressive symptoms and CVD indicating that both components of the depressive syndrome are relevant to cardiovascular outcomes. We further hypothesised that this association would be independent of known cardiovascular risk factors and that co-morbid anxiety would confer additive risk.

\section{Methods}

\subsection{Study design $\mathcal{E}$ sample}

This study was conducted in the context of The Irish LongituDinal study on Ageing (TILDA). TILDA recruited a stratified clustered sample of 8175 individuals representative of the community dwelling population aged 50 years and over in Ireland between October 2009 and February 2011. Wave 1 of the TILDA study included a face-to-face interview and a self-completion questionnaire, which was returned after the visit. The adjusted response rate to the study was $62 \%$ and response to the selfcompletion questionnaire was $84 \%$. Full details of the study sample and response rates have been described elsewhere (Kearney et al., 2011). Study participants were interviewed in their own homes by trained professional social interviewers who collected information regarding health, social and economic domains using Computer Assisted Personal Interviewing (CAPI). We applied a number of exclusion criteria. We excluded study participants with incomplete depression rating scores $(n=120)$. We also excluded study participants with self-reported cardiovascular disease not supported by use of cardiovascular medication $(n=166)$. We excluded patients who reported MI $(n=9)$, stroke $(n=6)$ or TIA $(n=14)$ within the previous 6 months to minimise the possibility that depressive symptoms had occurred as part of an adjustment reaction to a recent cardiovascular event. This left a sample of 7872 for analysis. Study participants gave informed consent and ethics approval was obtained from the faculty of health sciences research ethics committee of Trinity College Dublin.

\subsection{Measures}

\subsubsection{Socio-demographic characteristics}

Socio-demographic characteristics included age, gender, and education. Education was determined according to the highest educational level attained and was divided into three categories: completion of primary level education or lower, completion or part completion of secondary level education (defined as having completed intermediate or leaving certificate examinations) and third level education or higher (defined as having completed a diploma, primary or higher degree).

\subsubsection{Depressive symptoms}

Depressive symptoms were measured with the 20-item Center for Epidemiologic Studies Depression (CES-D) scale which assesses depressive symptoms over the previous week (Radloff, 1977). Each symptom is scored on a scale from 0 (rarely or none of the time, less than one day) to 3 (most or all of the time, five-seven days) to give a possible total score of 60 . Depression was defined as a score of $\geq 16$. A cut point of $\geq 16$ has been associated with $100 \%$ sensitivity and $88 \%$ specificity for major depression in community dwelling older adults (Beekman et al., 1997). The overlap with symptoms of physical illness has been shown to be very limited and false positives have not been found to be more likely among older adults with physical illness, cognitive decline or anxiety (Beekman et al., 1997; Berkman et al., 1986).

\subsubsection{Anxiety symptoms}

Anxiety symptoms were measured with the anxiety subscale of the Hospital Anxiety and Depression Scale (HADS-A). This is a self-report questionnaire, which assesses the frequency of anxiety symptoms over the last week. It contains 7 items rated on a fourpoint scale from 0 (not at all) to 3 (very often indeed) to give a possible maximum score of 21 . This scale was included in the selfreport questionnaire, which was returned by post after the initial interview $(n=6392)$. Anxiety was defined as a score of $\geq 8$. A cut point of $\geq 8$ has previously been reported to provide the optimal balance between sensitivity (0.89) and specificity (0.75) for generalised anxiety disorder (Bjelland et al., 2002; Olsson et al., 2005). 


\subsubsection{Cardiovascular disease}

During the interview, participants were asked if a doctor had ever told them that they had any of the following diagnoses: angina, heart attack, stroke, ministroke or Transient Ischaemic Attack/TIA. CVD was subdivided into cerebrovascular disease (stroke or TIA) and coronary vascular disease (angina pectoris or myocardial infarction). CVD was only considered present when self-report of the disease was supported by use of cardiovascular medication. This might be considered a conservative approach given that patients diagnosed with CVD several years previously may have stopped taking cardiovascular medication in the interim. Researchers obtained an inhome inventory of medication regularly used by direct observation and we included a large number of cardiovascular medications to minimise the possibility that patients with CVD might be inappropriately excluded for this reason. Medication was classified according to the World Health Organization Anatomical Therapeutic Chemical (ATC) classification (WHO, 2007). Medication for CVD included anticoagulant (ATC-code B01AA) or antiplatelet agents (B01AC) (N02BA01) or any medication for hypertension (antihypertensives [C02], diuretics [C03], beta blocking agents [C07], calcium channel blockers [C08], agents acting on reninangiotensin system [C09]), nitrate vasodilators [C01DA] or lipid modifying agents (C10). When self-report was not confirmed by medication use, CVD was considered to be 'undetermined' and subjects were excluded from the analyses $(n=166)$.

\subsubsection{Cardiovascular risk factors}

Participants were asked if they had ever received a diagnosis of diabetes mellitus, high cholesterol or hypertension from a doctor. Participants were asked if they had ever smoked in the past or were current smokers. Frequency of alcohol consumption was recorded on a scale from one (almost every day) to seven (not at all in the last 6 months). Physical activity was assessed using the short form of the International Physical Activity Questionnaire (IPAQ) (Craig et al., 2003). The IPAQ classified subjects as undertaking low, moderate or high levels of physical activity.

\subsection{Analysis}

Socio-demographic and clinical characteristics were compared between study participants with and without CVD using independent $t$-tests for continuous variables and chi-square statistics for categorical variables as appropriate. Logistic regression analyses were conducted to determine the association between depression and CVD before and after adjustment for cardiovascular risk factors. The consistency of the association between depression and CVD was checked by conducting separate logistic regression analyses for cerebrovascular disease (stroke and TIA) and coronary heart disease (angina pectoris and myocardial infarction). We investigated the association between anxiety and CVD in logistic regression analyses to determine if anxiety was independently predictive of CVD. We conducted an exploratory factor analysis to determine the underlying factors of the CES-D and their relationships with CVD. We then explored the associations between individual depressive symptoms and CVD before and after adjustment for relevant socio-demographic characteristics (age, gender, education) and cardiovascular risk factors (diabetes, hypertension, raised cholesterol, smoking, alcohol, physical activity). Depressive symptoms were entered both as dichotomous (present/absent, score of $\geq 1$ ) and continuous (score of $0,1,2 / 3$ ) variables. A standard significance level of $\alpha=0.05$ was used for most comparisons. In instances of multiple testing (i.e., CES-D scale items) a more conservative Bonferroni significance level of $0.05 / 20(p<0.0025)$ may be applied. Both unadjusted $p$ values and indicators of statistical significance following
Bonferroni adjustment are presented in tables for ease of interpretation. All statistical analyses were conducted with SPSS for windows version 18

\section{Results}

The mean age of the study sample $(n=7872)$ was $63.7(S D=9.7$, range 50-99). 4271 (54.3\%) of study participants were female. A total of $542(6.9 \%)$ study participants reported previous coronary vascular disease and 198 (2.5\%) reported previous cerebrovascular disease to give a total of 687 (8.7\%) of participants with any cardiovascular disease (CVD). A total of 738 (9.4\%) study participants reported depression (CES-D $\geq 16$ ). 1542 (24.1\% of returned anxiety scales) reported anxiety (HADS-A $\geq 8$ ). Participants with milder depressive symptoms were more likely to return the anxiety questionnaire $(5.6+/-6.9$ vs $6.7+/-7.9, d f=7870, t=5.3$, $p<0.001)$. Socio-demographic and clinical characteristics of the study population according to presence or absence of any cardiovascular disease (CVD) are outlined in Table 1 . Depression was associated with a $60 \%, 90 \%$ and $80 \%$ increased risk of coronary vascular disease, cerebrovascular disease and CVD, respectively following adjustment for socio-demographic and cardiovascular risk factors (age, gender, education, smoking, physical activity, alcohol intake, raised cholesterol, hypertension and diabetes) (Table 2). Depressive symptoms were similarly associated with increased risk of coronary vascular disease, cerebrovascular disease and CVD both before and after adjustment for risk factors.

The relationship between both depression and CVD (OR 1.64, 95\% CI 1.2-2.3, $p=0.002$ ) and depressive symptoms and CVD (OR 1.02 95\% CI 1.01-1.04, $p=0.009$ ) remained significant following further adjustment for anxiety (HADS-A $\geq 8$ ) and anxiety symptoms, respectively indicating a $2 \%$ increased risk of CVD for each additional point on the CES-D scale and a $64 \%$ increased risk for depression. Anxiety (OR 1.48, 95\% CI 1.18-1.85, $p=0.001$ ) and anxiety symptoms (OR 1.03, 95\% CI 1.007-1.06, $p=0.014$ ) were significantly associated with CVD following adjustment for sociodemographic characteristics and cardiovascular risk factors.

The relationship between anxiety symptoms and CVD was no longer significant following further adjustment for depressive symptoms (OR $1.02,95 \%$ CI $0.98-1.05, p=0.34$ ) while anxiety remained independently associated with CVD following further adjustment for depression (OR 1.31, 95\% CI 1.03-1.67, $p=0.026$ ). 389 (70.9\%) participants with depression (who had also completed the HADS-A) reported anxiety and the mean CES-D score among these participants was greater than that observed among depressed participants without anxiety $(24.4+/-7.8$ vs $20.5+/-4.9, d f=547, t=-5.8, p<0.001)$. However, depressed participants with co-morbid anxiety were not at significantly increased risk of CVD compared to those with depression alone (OR 1.1, 95\% CI 0.6-2.1, $p=0.75$ ).

Table 1

Population characteristics according to presence or absence of cardiovascular disease (CVD)

\begin{tabular}{lllll}
\hline & $\begin{array}{l}\text { CVD } \\
(n=687)\end{array}$ & $\begin{array}{l}\text { No CVD } \\
(n=7185)\end{array}$ & $x_{/ t \text { value }}^{2} p$ & \\
\hline Age, mean (SD) & $70.5(9.0)$ & $63.0(9.5)$ & -20.6 & $<0.001$ \\
Male (\%) & $425(62)$ & $3176(44)$ & 78.8 & $<0.001$ \\
Educational level (Primary, \%) & $312(45)$ & $2061(29)$ & 85.9 & $<0.001$ \\
Smoking status (Never, \%) & $203(30)$ & $3251(45)$ & 81.5 & $<0.001$ \\
Alcohol (Almost every day, \%) & $50(7.3)$ & $365(5.1)$ & 45.2 & $<0.001$ \\
Physical Activity (level 0) & $281(41)$ & $2177(30.3)$ & 49.4 & $<0.001$ \\
Diabetes (\%) & $98(14.3)$ & $503(7.0)$ & 46.9 & $<0.001$ \\
Hypertension (\%) & $424(61.7)$ & $2463(34.3)$ & 203.3 & $<0.001$ \\
Raised Cholesterol (\%) & $393(57.2)$ & $2580(35.9)$ & 121.0 & $<0.001$ \\
Depression (CES-D $\geq 16)$ & $93(13.5)$ & $645(9.0)$ & 15.2 & $<0.001$ \\
\hline
\end{tabular}


Table 2

Association between depressive symptoms and depression according to type of cardiovascular disease.

\begin{tabular}{|c|c|c|c|c|c|c|}
\hline & OR & $95 \% \mathrm{CI}$ & $p$ & OR & $95 \% \mathrm{CI}$ & $p^{*}$ \\
\hline \multicolumn{7}{|l|}{ Depressive symptoms } \\
\hline Coronary vascular disease & 1.02 & $1.01-1.03$ & 0.004 & 1.02 & $1.01-1.03$ & 0.014 \\
\hline Cerebro-vascular disease & 1.03 & $1.01-1.05$ & $<0.001$ & 1.03 & $1.01-1.05$ & 0.005 \\
\hline Any cardiovascular disease & 1.02 & $1.01-1.03$ & $<0.001$ & 1.02 & $1.01-1.03$ & 0.001 \\
\hline \multicolumn{7}{|l|}{ Depression $(C E S-D \geq 16)$} \\
\hline Coronary vascular disease & 1.5 & $1.1-1.9$ & 0.004 & 1.6 & $1.2-2.3$ & 0.004 \\
\hline Cerebro-vascular disease & 1.9 & $1.3-2.8$ & 0.001 & 1.9 & $1.3-3.1$ & 0.003 \\
\hline Any cardiovascular disease & 1.6 & $1.3-2.0$ & $<0.001$ & 1.8 & $1.3-2.3$ & $<0.001$ \\
\hline
\end{tabular}

* Adjusted for age, gender, education, smoking, physical activity, alcohol intake, raised cholesterol, hypertension and diabetes.

Table 3

Relationship between symptom factors and cardiovascular disease (CVD).

\begin{tabular}{|c|c|c|c|c|c|c|}
\hline & OR & $95 \% \mathrm{CI}$ & $p$ & OR & $95 \% \mathrm{CI}$ & $p^{*}$ \\
\hline Depressed Affect/Somatic complaints & 1.05 & $1.03-1.08$ & $<\mathbf{0 . 0 0 1}$ & 1.06 & $1.03-1.09$ & $<0.001$ \\
\hline Positive Affect & 1.04 & $0.98-1.11$ & 0.163 & 1.05 & $0.97-1.13$ & 0.227 \\
\hline Inter-personal Problems & 1.06 & $0.89-1.26$ & 0.532 & 1.16 & $0.94-1.44$ & 0.168 \\
\hline
\end{tabular}

* Adjusted for age, gender, education, smoking, physical activity, alcohol intake, raised cholesterol, hypertension and diabetes.

Table 4

Proportion of subjects reporting each depressive symptom (dichotomous variable) according to presence or absence of cardiovascular disease.

\begin{tabular}{|c|c|c|c|c|c|c|}
\hline & $\begin{array}{l}\text { CVD } \\
(n \%)\end{array}$ & $\begin{array}{l}\text { No CVD } \\
(n \%)\end{array}$ & OR & $95 \% \mathrm{CI}$ & $p^{+}$ & $p^{*}$ \\
\hline \multicolumn{7}{|l|}{ Depressed Affect/Somatic complaints } \\
\hline 1. Bothered by things. & $161(23.4)$ & $1400(19.5)$ & 1.6 & $1.3-1.9$ & $<0.001^{\mathrm{b}}$ & $0.001^{b}$ \\
\hline 2. Appetite was poor. & $109(15.9)$ & $791(11.0)$ & 1.6 & $1.2-1.9$ & $<0.001^{\mathrm{b}}$ & 0.018 \\
\hline 3. Could not shake off the blues. & $114(16.6)$ & $890(12.4)$ & 1.7 & $1.3-2.1$ & $<\mathbf{0 . 0 0 1}^{\mathrm{b}}$ & 0.004 \\
\hline 5. Trouble keeping my mind.. & $207(30.1)$ & $1830(25.5)$ & 1.5 & $1.2-1.8$ & $<0.001^{\mathrm{b}}$ & 0.008 \\
\hline 6. I felt depressed. & $165(24.0)$ & 1199(16.7) & 1.8 & $1.5-2.1$ & $<\mathbf{0 . 0 0 1}^{\mathrm{b}}$ & $0.001^{b}$ \\
\hline 7. Everything was an effort. & $193(28.1)$ & $1409(19.6)$ & 1.7 & $1.4-2.0$ & $<0.001^{\mathrm{b}}$ & 0.008 \\
\hline 9. I thought my life had been a failure. $71(10.3)$ & & $609(8.5)$ & 1.5 & $1.1-1.9$ & 0.004 & 0.030 \\
\hline 10. I felt fearful. & $112(16.3)$ & $1055(14.7)$ & 1.4 & $1.1-1.7$ & 0.006 & 0.022 \\
\hline 11. Sleep was restless. & $294(42.8)$ & 2851(39.7) & 1.4 & $1.1-1.6$ & $<\mathbf{0 . 0 0 1}^{\mathrm{b}}$ & 0.37 \\
\hline 13. Talked less than usual. & $117(17.0)$ & $1076(15.0)$ & 1.3 & $1.0-1.6$ & 0.042 & 0.47 \\
\hline 14. I felt lonely. & $162(23.6)$ & $1317(18.3)$ & 1.4 & $1.2-1.7$ & $<\mathbf{0 . 0 0 1}^{\mathrm{b}}$ & 0.035 \\
\hline 17. I had crying spells. & $79(11.5)$ & $779(10.8)$ & 1.5 & $1.1-1.9$ & 0.005 & 0.026 \\
\hline 18. I felt sad. & $219(31.9)$ & $1813(25.2)$ & 1.6 & $1.3-1.9$ & $<\mathbf{0 . 0 0 1}^{\mathrm{b}}$ & $<\mathbf{0 . 0 0 1}^{\mathrm{b}}$ \\
\hline 20. Could not get going. & $162(23.6)$ & $1348(18.8)$ & 1.5 & $1.2-1.8$ & $<\mathbf{0 . 0 0 1}^{\mathrm{b}}$ & 0.008 \\
\hline \multicolumn{7}{|l|}{ Positive Affect } \\
\hline 4. Felt as good as other people. & $126(18.3)$ & $1263(17.6)$ & 1.1 & $0.87-1.3$ & 0.52 & 0.91 \\
\hline 8. Felt hopeful about the future. & $275(40.0)$ & $2577(35.9)$ & 1.2 & $0.99-1.4$ & 0.06 & 0.27 \\
\hline 12. I was happy. & $209(30.4)$ & $2167(30.2)$ & 1.1 & $0.95-1.4$ & 0.16 & 0.27 \\
\hline 16. I enjoyed life. & $181(26.3)$ & $1749(24.4)$ & 1.2 & $1.03-1.5$ & 0.023 & 0.23 \\
\hline \multicolumn{7}{|l|}{ Interpersonal Problems } \\
\hline 15. People were unfriendly. & $62(9.0)$ & $614(8.6)$ & 1.2 & $0.91-1.6$ & 0.19 & 0.42 \\
\hline 19. Felt that people dislike me. & $47(6.8)$ & $447(6.2)$ & 1.5 & $1.07-2.1$ & 0.018 & 0.13 \\
\hline
\end{tabular}

+ Adjusted for age, gender and education.

* Adjusted for age, gender, education, smoking, physical activity, alcohol intake, raised cholesterol, hypertension and diabetes.

b Remains significant at Bonferroni adjusted significance level of $p<0.0025$.

We then conducted an exploratory factor analysis to determine whether certain factors of the CES-D were more closely associated with CVD. Based on a scree plot and the criterion of Eigenvalue $>1$, we determined the optimal number of factors to be three, which together accounted for $44.8 \%$ of the variance (Table 3 ). Factor 1 (Depressed Affect/Somatic Complaints factor) contained a combination of the items from the Depressed Affect and Somatic and Retarded Activity factors as originally described by Radloff (1977). Factors 2 and 3 replicated the Positive Affect and Interpersonal Problems factors which have previously been described (Shafer, 2006). The symptoms included in each of these factors are presented in Tables 4 and 5 . Several symptoms from factor 1 (Depressed Affect/Somatic Complaints factor) displayed a significant association with CVD following adjustment for socio-demographic characteristics. The sum score of symptoms from factor 1 was significantly associated with increased risk of CVD and we observed a $6 \%$ increased risk for each additional symptom endorsed. Study participants who endorsed just two or more symptoms from factor 1 had a mean CES-D score of $10.4(S D=7.8)$ and displayed a $21 \%$ increased risk of CVD following adjustment for socio-demographic characteristics and cardiovascular risk factors (OR 1.21 95\% CI 1.01$1.5, p=0.04$ ). Participants who endorsed five or more symptoms had a mean score of $16.2(S D=8.2)$ and displayed a $64 \%$ increased risk following adjustment (OR $1.6495 \%$ CI 1.3-2.0, $p<0.001$ ). A similar dose-response relationship was not observed for the other two factors.

The association between each depressive symptom entered as a dichotomous variable (present/absent, score of $\geq 1$ ) and CVD is 
Table 5

Proportion of subjects reporting each depressive symptom (continuous variable, score $0,1,2 / 3$ ) according to presence or absence of cardiovascular disease.

\begin{tabular}{|c|c|c|c|c|c|c|}
\hline & OR & $95 \% \mathrm{CI}$ & $p^{+}$ & OR & $95 \% \mathrm{CI}$ & $p^{*}$ \\
\hline \multicolumn{7}{|l|}{ Depressed Affect/Somatic complaints } \\
\hline 1. Bothered by things. & 1.07 & $1.06-1.08$ & $<\mathbf{0 . 0 0 1}^{\mathrm{b}}$ & 1.22 & $1.05-1.42$ & 0.011 \\
\hline 2. Appetite was poor. & 1.38 & $1.18-1.61$ & $<\mathbf{0 . 0 0 1}^{\mathrm{b}}$ & 1.26 & $1.05-1.50$ & 0.012 \\
\hline 3. Could not shake off the blues. & 1.40 & $1.15-1.59$ & $<\mathbf{0 . 0 0 1}^{\mathrm{b}}$ & 1.26 & $1.04-1.52$ & 0.016 \\
\hline 5. Trouble keeping my mind.. & 1.25 & $1.10-1.40$ & $0.001^{\mathrm{b}}$ & 1.17 & $1.01-1.35$ & 0.033 \\
\hline 6. I felt depressed. & 1.49 & $1.31-1.71$ & $<\mathbf{0 . 0 0 1}^{\mathrm{b}}$ & 1.29 & $1.11-1.52$ & $0.001^{b}$ \\
\hline 7. Everything was an effort. & 1.47 & $1.29-1.66<$ & $\mathbf{0 . 0 0 1}^{\mathrm{b}}$ & 1.25 & $1.08-1.45$ & $0.002^{b}$ \\
\hline 9. I thought my life had been a failure. & 1.28 & $1.08-1.53$ & 0.006 & 1.21 & $0.99-1.49$ & 0.07 \\
\hline 10. I felt fearful. & 1.23 & $1.05-1.45$ & 0.01 & 1.20 & $1.02-1.46$ & 0.03 \\
\hline 11. Sleep was restless. & 1.24 & $1.12-1.37$ & $<\mathbf{0 . 0 0 1}^{\mathrm{b}}$ & 1.09 & $0.97-1.22$ & 0.16 \\
\hline 13. Talked less than usual. & 1.12 & $1.02-1.38$ & 0.026 & 1.08 & $0.91-1.29$ & 0.37 \\
\hline 14. I felt lonely. & 1.22 & $1.07-1.39$ & $0.002^{\mathrm{b}}$ & 1.13 & $0.98-1.32$ & 0.10 \\
\hline 17. I had crying spells. & 1.26 & $1.04-1.51$ & 0.018 & 1.20 & $0.97-1.49$ & 0.09 \\
\hline 18. I felt sad. & 1.41 & $1.24-1.59$ & $<\mathbf{0 . 0 0 1}^{\mathrm{b}}$ & 1.34 & $1.16-1.54$ & $<\mathbf{0 . 0 0 1}^{\mathrm{b}}$ \\
\hline 20. Could not get going. & 1.40 & $1.22-1.61$ & $<\mathbf{0 . 0 0 1}^{\mathrm{b}}$ & 1.29 & $1.09-1.50$ & $0.002^{b}$ \\
\hline \multicolumn{7}{|l|}{ Positive Affect } \\
\hline 4. Felt as good as other people. & 1.04 & $0.92-1.17$ & 0.55 & 0.98 & $0.85-1.13$ & 0.78 \\
\hline 8. I felt hopeful about the future. & 1.12 & $1.02-1.24$ & 0.02 & 1.07 & $0.95-1.19$ & 0.26 \\
\hline 12. I was happy. & 1.05 & $0.93-1.12$ & 0.42 & 1.04 & $0.91-1.18$ & 0.56 \\
\hline 16. I enjoyed life. & 1.16 & $1.03-1.31$ & 0.01 & 1.09 & $0.95-1.25$ & 0.21 \\
\hline \multicolumn{7}{|l|}{ Interpersonal Problems } \\
\hline 15. People were unfriendly. & 1.12 & $0.92-1.37$ & 0.27 & 1.11 & $0.88-1.39$ & 0.38 \\
\hline 19. I felt that people dislike me. & 1.34 & $1.09-1.71$ & 0.005 & 1.32 & $1.02-1.71$ & 0.03 \\
\hline
\end{tabular}

+ Adjusted for age, gender and education.

* Adjusted for age, gender, education, smoking, physical activity, alcohol intake, raised cholesterol, hypertension and diabetes.

${ }^{\mathrm{b}}$ Remains significant at Bonferroni adjusted significance level of $p<0.0025$.

outlined in Table 4. Similarly, the association between each depressive symptom entered as a continuous variable (score of $0,1,2 / 3$ ) and CVD is outlined in Table 5. A number of depressive symptoms from factor 1 displayed independent associations with CVD. These included core aspects of the depressive syndrome which might be considered to be both cognitive and somatic in nature. All participants with depression endorsed at least one of these symptoms. Positive affective and interpersonal items displayed less robust associations with CVD and none were statistically significant following application of a stricter Bonferroni adjusted significance level $(p<0.0025)$.

\section{Discussion}

In this study we found a significant relationship between depressive symptoms and cardiovascular disease with a $2 \%$ increased risk of co-morbid CVD for each additional point on the 60-item CES-D scale. Participants with depression were $80 \%$ more likely to have co-morbid CVD independently of socio-demographic characteristics and cardiovascular risk factors. This is consistent with previous studies, which have reported comparable levels of increased risk with an evident dose response according to severity of depressive symptoms (Van Der Kooy et al., 2007; Rugulies, 2002). In particular, we found a consistent relationship between a number of core depressive symptoms, which loaded onto the same factor, and CVD. These symptoms related to low mood, sadness, amotivation, fatigue, diminished appetite and concentration difficulties and included aspects of the depressive syndrome traditionally considered to be both cognitive and somatic in nature. Each additional symptom from this factor was associated with a 6\% increased risk of CVD. In line with previous findings we found that, even at levels not ordinarily considered clinically significant (scores of approximately $\geq 10$ ), these depressive symptoms were independently associated with increased risk of CVD (Bush et al., 2001). We also found that anxiety was associated with increased risk of CVD although study participants with both depression and co-morbid anxiety were not at increased risk compared to those with depression alone.
With regard to the nature and content of individual symptoms, our findings are consistent with those previously reported for somatic symptoms (De Jonge et al., 2006; Hoen et al., 2010). In particular, fatigue and appetite problems have previously been reported to be independently predictive of incident cardiovascular events in outpatients with stable coronary heart disease (Hoen et al., 2010). Similarly these symptoms were included in a somatic factor, which was found to be predictive of further cardiovascular events in patients following myocardial infarction (De Jonge et al., 2006). We found that restless sleep was significantly associated with CVD but not following adjustment for risk factors. Early morning wakening and symptoms of autonomic arousal have previously been associated with cardiac disease in a crosssectional analysis (Fraguas et al., 2007). A prospective analysis of this item reported a more variable relationship with cardiac outcomes (Hoen et al., 2010). Interestingly, we also found that a range of depressive symptoms which might be best considered as cognitive in nature such as; low mood, sadness, feeling bothered by things, not being able to "shake off the blues" and to a lesser extent; thinking that one's life had been a failure, feeling lonely and having crying spells were associated with CVD. Of these, feeling depressed and sad were most consistently associated with CVD and remained significantly associated following application of a conservative Bonferroni correction. Depressed mood has previously been associated with increased risk of cardiovascular events but not following adjustment for risk factors (Hoen et al., 2010). Inadequate power may have accounted for the negative findings in this instance. A strong association between symptoms of sadness, crying and subsequent cardiac death has previously been reported (Barefoot et al., 2000). This association was found to be more predictive of mortality than somatic depressive symptoms. Similarly, sadness and crying were previously included in a "somatic/ affective" factor which was predictive of further cardiovascular events while negative cognitions such as self-dislike, guilt and a sense of failure were not found to be significantly predictive (De Jonge et al., 2006). The CES-D does not contain a number of cognitive items such as guilt or self-dislike. However, we found that thoughts of failure were significantly associated with CVD in most 
analyses in this study. Taken together, these findings suggest that certain core cognitive depressive symptoms such as low mood and sadness have been more consistently associated with CVD than other cognitive items. The observation that both cognitive and somatic symptoms of depression are associated with cardiovascular outcomes decreases the possibility that the association is simply secondary to residual confounding by physical symptoms from comorbid medical conditions. It is also noteworthy that while certain depressive symptoms remained significantly associated with CVD following adjustment for cardiovascular risk factors, others did not. This suggests some variability in the mechanisms of association between individual symptoms and CVD. This would have important implications for interventional strategies.

We found that anxiety was associated with increased risk of CVD, which is consistent with previous findings suggesting that both anxiety and depression are relevant to cardiac outcomes (Roest et al., 2010; Martens et al., 2010). However, in contrast to our hypothesis, we found that participants with depression and co-morbid anxiety were not at increased risk compared to those with depression alone. This has previously been reported (Frasure-Smith and Lesperance, 2008) although other investigators using different methodology in a psychopathology-based cohort found that anxiety accounted for most the association between depression and CVD (Vogelzangs et al., 2010). Anxiety is closely linked to depression and generalised anxiety disorder may precede depression in up to one third of cases and vice versa (Moffitt et al., 2007). The CES-D contains a number of items that are relevant to anxiety such as concentration difficulties, sleep disturbance and fearfulness and there is some overlap in this regard. We found that participants with co-morbid anxiety were more severely depressed but that the magnitude of this increase was not sufficiently large to impact upon cardiovascular risk. The relationship between the positive affective and interpersonal items and CVD was more variable. It has previously been reported that positive affect may protect against incident cardiovascular events (Davidson et al., 2010). We found that two positive affective items which referred to hope and enjoyment were associated with CVD but not following adjustment for cardiovascular risk factors. One of the CES-D items, which refers to interpersonal functioning ("I felt that people dislike me") displayed a significant association with CVD but not following Bonferroni correction. Interpersonal sensitivity is a well described feature of atypical depression which has in turn been associated with increased sleep and weight gain (Pae et al., 2009). However, we were unable to formally diagnose atypical depression in this analysis and so we may only speculate regarding the possible associations in this regard.

This study has a number of strengths and limitations. Strengths include the large study sample representative of the community dwelling population age 50 years and over. This allowed greater power to investigate the relationship between individual symptoms and CVD than permitted in previous analyses. In addition, we conducted a detailed assessment of study participants and were able to adjust for several socio-demographic and cardiovascular risk factors. Limitations include the cross-sectional design, which precludes conclusions regarding causality, and it is possible that the depressive symptoms observed occurred as a consequence of cardiovascular disease. We did however try to reduce this possibility by excluding the small proportion of patients who had experienced a cardiovascular event within the previous 6 months. Depression was assessed by self-report rather than diagnostic interview although the CES-D is a well validated and frequently used measure in older adults. A proportion of study participants with greater depressive symptoms did not return the anxiety questionnaire and so we cannot draw definitive conclusions regarding the relative associations between anxiety, depression and CVD in this subgroup. In addition, testing the associations between individual depressive symptoms and CVD necessarily involves multiple testing thus inflating the possibility for type I errors. These results should therefore be interpreted with this caveat in mind although it is noteworthy that there is a certain consistency to the findings reported that many significant associations persist following application of a conservative Bonferroni correction. Of course, findings from this sample of community dwelling older adults may not be readily generalised to younger populations. Similarly, it has been proposed that depression in the immediate aftermath of an acute coronary syndrome may be qualitatively distinct from that observed in community populations and so findings may not be readily generalised to inpatient populations (Poole et al., 2011).

In conclusion, we found a strong and consistent relationship between depression and CVD. This relationship was independent of socio-demographic and cardiovascular risk factors. In particular, we found that a number of core depressive symptoms which related to low mood, sadness, amotivation, fatigue, diminished appetite and concentration difficulties were consistently associated with CVD in a dose-related fashion. These symptoms include aspects of the depressive syndrome traditionally considered to be both cognitive and somatic in nature. Symptoms relating to interpersonal problems and positive affect were less consistently associated with CVD. Co-morbid anxiety did not confer additive risk in participants with depression. This suggests that clinicians should give due regard to both cognitive and somatic aspects of the depressive syndrome when determining cardiovascular risk. Future longitudinal investigation should confirm these findings and explore potential pathological mechanisms.

\section{Role of funding source}

Funding for The Irish LongituDinal study of Ageing (TIDLA) was provided by the Department of Health, Irish Life and the Atlantic Philanthropies.

\section{Conflict of interest}

None.

\section{Acknowledgement}

Funding for The Irish LongituDinal study of Ageing (TILDA) was provided by the Department of Health, Irish Life and the Atlantic Philanthropies.

\section{References}

Barefoot, J.C., Brummett, B.H., Helms, M.J., Mark, D.B., Siegler, I.C., Williams, R.B. 2000. Depressive symptoms and survival of patients with coronary artery disease. Psychosomatic Medicine 62, 790-795.

Barth, J., Schumacher, M., Herrmann-Lingen, C., 2004. Depression as a risk factor for mortality in patients with coronary heart disease: a meta-analysis. Psychosomatic Medicine 66, 802-813.

Beekman, A.T., Deeg, D.J., Van Limbeek, J., Braam, A.W., De Vries, M.Z., Van Tilburg, W., 1997. Criterion validity of the center for epidemiologic studies depression scale (CES-D): results from a community-based sample of older subjects in The Netherlands. Psychological Medicin 27, 231-235.

Berkman, L.F., Berkman, C.S., Kasl, S., Freeman, D.H.J., Leo, L., Ostfeld, A.M., Cornoni-Huntley, J., Brody, J.A., 1986. Depressive symptoms in relation to physical health and functioning in the elderly. American Journal of Epidemiology $124,372-388$.

Bjelland, I., Dahl, A.A., Haug, T.T., Neckelmann, D., 2002. The validity of the hospital anxiety and depression scale. An updated literature review. Journal of Psychosomatic Research 52, 69-77.

Brodaty, H., Cullen, B., Thompson, C., Mitchell, P., Parker, G., Wilhelm, K., Austin, M.P., Malhi, G., 2005. Age and gender in the phenomenology of depression. American Journal of Geriatric Psychiatry 13, 589-596.

Bush, D.E., Ziegelstein, R.C., Tayback, M., Richter, D., Stevens, S., Zahalsky, H., Fauerbach, J.A., 2001. Even minimal symptoms of depression increase mortality risk after acute myocardial infarction. American Journal of Cardiology 88 337-341.

Craig, C.L., Marshall, A.L., Sjostrom, M., Bauman, A.E., Booth, M.L., Ainsworth, B.E. Pratt, M., Ekelund, U., Ynqve, A., Sallis, J.F., OJA, P., 2003. International physical activity questionaire: 12-country reliability and validity. Medicine and Science in Sports and Exercise 35, 1381-1395. 
Davidson, K.W., Mostofsky, E., Whang, W., 2010. Do not worry, be happy: positive affect and reduced 10-year incident coronary heart disease: the Canadian Nova Scotia Health Survey. European Heart Journal 31, 1065-1070.

De Jonge, P., Ormel, J., Van Den Brink, R.H., Van Melle, J.P., Spijkerman, T.A. Kuijper, A., Van Veldhuisen, D.J., Van Den Berg, M.P., Honig, A., Crijns, H.J. Schene, A.H., 2006. Symptom dimensions of depression following myocardial infarction and their relationship with somatic health status and cardiovascular prognosis. American Journal of Psychiatry 163, 138-144.

Fraguas Jr., R., Iosifescu, D.V., Alpert, J., Wisniewski, S.R., Barkin, J.L., Trivedi, M.H. Rush, A.J., Fava, M., 2007. Major depressive disorder and comorbid cardiac disease: is there a depressive subtype with greater cardiovascular morbidity? Results from the STAR*D study. Psychosomatics 48, 418-425.

Frasure-Smith, N., Lesperance, F., 2008. Depression and anxiety as predictors of 2 year cardiac events in patients with stable coronary artery disease. Archives of General Psychiatry 65, 62-71.

Frasure-Smith, N., Lesperance, F., 2010. Depression and cardiac risk: present status and future directions. Postgraduate Medical Journal 86, 193-196.

Gallagher, D., 2007. Depression and cardiovascular disease: does antidepressant treatment improve cardiac outcome? Irish Journal of Psychological Medicine 24, 156-159.

Hoen, P.W., Whooley, M.A., Martens, E.J., Na, B., Van Melle, J.P., De Jonge, P., 2010 Differential associations between specific depressive symptoms and cardiovascular prognosis in patients with stable coronary heart disease. Journal of the American College of Cardiology 56, 838-844.

Joynt, K.E., Whellan, D.J., O'connor, C.M., 2003. Depression and cardiovascular disease: mechanisms of interaction. Biological Psychiatry 54, 248-261.

Kearney, P.M., Cronin, H., O'regan, C., Kamiya, Y., Savva, G.M., Whelan, B., Kenny, R., 2011. Cohort profile: the Irish longitudinal study on ageing. International Journal of Epidemiology 40, 877-884.

Kessing, L.V., 2007. Epidemiology of subtypes of depression. Acta Psychiatrica Scandinavica 115, 85-89.

Licht-Strunk, E., Bremmer, M.A., Van Marwijk, H.W.J., Deeg, D.J.H., Hoogendijk, W.J.G., De Haan, M., Van Tilburg, W., Beekman, A.T.F., 2004. Depression in older persons with versus without vascular disease in the open population: similar depressive symptom patterns, more disability. Journal of Affective Disorders 83, 155-160.
Martens, E.J., De Jonge, P., Na, B., Cohen, B.E., Lett, H., Whooley, M.A., 2010. Scared to death? Generalized anxiety disorder and cardiovascular events in patients with stable coronary heart disease: the heart and soul study. Archives of General Psychiatry 67, 750-758.

Moffitt, T.E., Harrington, H., Caspi, A., Kim-Cohen, J., Goldberg, D., Gregory, A.M., Poulton, R., 2007. Depression and generalized anxiety disorder: cumulative and sequential comorbidity in a birth cohort followed prospectively to age 32 years. Archives of General Psychiatry 64, 651-660.

Olsson, I., Mykletun, A., Dahl, A.A., 2005. The hospital anxiety and depression rating scale: a cross-sectional study of psychometrics and case finding abilities in general practice. BMC Psychiatry 5, 46.

Pae, C.U., Tharwani, H., Marks, D.M., Masand, P.S., Patkar, A.A, 2009. Atypical depression: a comprehensive review. CNS Drugs 23, 1023-1037.

Poole, L., Dickens, C., Steptoe, A., 2011. The puzzle of depression and acute coronary syndrome: reviewing the role of acute inflammation. Journal of Psychosomatic Research 71, 61-68.

Radloff, L.S., 1977. The CES-D scale: a self-report depression scale for research in the general population. Applied Psychological Measurement 1, 385-401.

Roest, A.M., Martens, E.J., De Jonge, P., Denollet, J., 2010. Anxiety and risk of incident coronary heart disease: a meta-analysis. Journal of the American College of Cardiology 56, 38-46.

Rugulies, R., 2002. Depression as a predictor for coronary heart disease. a review and meta-analysis. American Journal of Preventive Medicine 23, 51-61.

Shafer, B.A., 2006. Meta-analysis of the factor structures of four depression questionnaires: beck, CES-D, Hamilton and Zung. Journal of Clinical Psychology 62, 123-146.

Van Der Kooy, K., Van Hout, H., Marwijk, H., Marten, H., Stehouwer, C., Beekman, A., 2007. Depression and the risk for cardiovascular diseases: systematic review and meta analysis. International Journal of Geriatric Psychiatry 22 613-626.

Vogelzangs, N., Seldenrijk, A., Beekman, A.T., Van Hout, H.P., De Jonge, P., Penninx, B.W., 2010. Cardiovascular disease in persons with depressive and anxiety disorders. Journal of Affective Disorders 125, 241-248.

WHO (2007) WHO Collaborating Centre for Drug Statistics Methodology. 